\title{
Beta-endorphin levels in both painful and painless diabetic peripheral neuropathy and its relations to pain characters and severity
}

\section{ABSTRACT}

Introduction. In the peripheral nervous system (PNS), $\beta$-endorphins produce analgesia by binding to opioid receptors (particularly of the mu subtype) at both preand post-synaptic nerve terminals, primarily exerting their effect through presynaptic binding.

Aim was to study serum $\beta$-endorphin levels in diabetic patients with and without diabetic peripheral neuropathy and its relations to characters and severity of pain in patients with painful diabetic peripheral neuropathy. Material and methods. The study was a case control study including 88 participants; 73 diabetics and 15 age and sex matched healthy subjects. For all subjects, levels of $\mathrm{HbA}_{1 \mathrm{c}}$, serum creatinine, total cholesterol, triglycerides, $\mathrm{HDL}$ and $\mathrm{LDL}$ as well as serum levels of $\beta$-endorphin were measured. Pain severity was detected by using visual analogue pain scale.

Results. Serum $\beta$-endorphin shows no significant difference between diabetic neuropathic, diabetic non neuropathic and control groups $(p=0.275)$. Serum $\beta$-endorphin shows negative correlation with age $(p=0.049)$ and $\mathrm{HbA}_{1 \mathrm{c}}(\mathrm{p}=0.048)$. While it was not correlated with pain severity $(p=0.371)$, NDS: total score $(p=0.803)$, BMI $(p=0.801)$, serum creatinine $(p=0.074)$ or DM duration ( $p=0.607)$. Serum $\beta$-endorphin shows

Address for correspondence:

Enas T. Elkhamisy

Internal Medicine Department, Faculty of Medicine

Mansoura University, Egypt

e-mail: enaselkhamisy@yahoo.com

Clinical Diabetology 2017, 6, 5, 159-171

DOI: $10.5603 /$ DK.2017.0027

Received: 28.07.2017

Accepted: 09.10.2017 no significant difference between painful and painless neuropathy subgroups ( $p=0.701$ ).

Conclusion. In our study serum $\beta$-endorphin levels showed no significant difference between patients with painless diabetic peripheral neuropathy and those with painful diabetic peripheral neuropathy with different characters of pain. Also, serum $\beta$-endorphin levels was not correlated with pain severity. (Clin Diabetol 2017; 6, 5: 159-171)

Key words: endorphin, diabetes, neuropathy

\section{Introduction}

Diabetic peripheral neuropathy (DPN) is a common, distressing and debilitating complication that develops in up to $30-50 \%$ of diabetic patients. Distal symmetric polyneuropathy (DSP) is the commonest type of neuropathy complicating diabetes [1]. Pain is the most agonizing symptom of DPN [2].

The pathogenesis of DPN is not completely understood, however vascular and metabolic factors are incriminated [3].

Magnetic resonance imaging (MRI) detected shrinkage of the spinal cord in patients with DPN [4], and also dysregulations of the somatosensory afferent pathways, thalamic neuronal dysfunction, and perfusion abnormalities, which reports a significant involvement of the CNS in patients with DPN [3].

Beta-endorphins are neuropeptides that have morphine like effects [5]. They are synthesized and stored in the anterior pituitary gland from their precursor protein ProOpioMelanoCortin (POMC), that is synthesized in response to a signal from the hypothalamus; 
which is corticotrophic-releasing hormone (CRH), released in response to physiologic stress as pain, and postoperative [6].

Beta-endorphins produce analgesia in the peripheral nervous system (PNS), by binding to opioid receptors (particularly of the mu subtype) at both pre- and post-synaptic nerve terminals, resulting in inhibition of the release of tachykinins, particularly substance $P$, preventing the transmission of pain [7].

In the CNS, beta-endorphins similarly bind muopioid receptors and exert their primary action at presynaptic nerve terminals, they exert their analgesic effect by inhibiting the release of gamma-aminobutyric acid (GABA), an inhibitory neurotransmitter, resulting in excess production of dopamine, which is associated with pleasure [8], and patients with chronic neuropathic pain were found to have low levels of $\beta$-endorphin in the cerebrospinal fluid (CSF), which indicates a defective down modulation of pain in chronic neuropathic pain [9].

The aim of this work was to study serum $\beta$-endorphin levels in diabetic patients with and without diabetic peripheral neuropathy and to study the possible differences between patients with painful and painless diabetic peripheral neuropathy. We also aimed to study serum $\beta$-endorphin levels relations to characters and severity of pain in patients with painful diabetic peripheral neuropathy.

\section{Material and methods}

The study included 88 participants, 73 of them were diabetics (cases) recruited from Mansoura Specialized Medical Hospital (diabetes outpatient clinics) from February 2015 to December 2015, and 15 participants were age and sex matched healthy subjects (control group). Diabetic state was confirmed or excluded according to the revised American Diabetes Association criteria [10]. Ethical approval was obtained and each subject gave a written informed consent. The patients were divided into three groups Group (1); 57 diabetic subject with DPN which were further subdivided into 2 subgroups according to presence or abscence of pain. Visual analogue pain scale was used to assess the severity of pain. Group (2); 16 diabetic subjects without peripheral neuropathy. Group (3); control group consists of 15 healthy subjects.

Diagnosis of DPN was based on modified neuropathy disability score. We use score $\geq 6$ as a cut point for diagnosis of DPN. Presence of pain in the legs was known from history and its severity was graded according to visual analogue pain scale. The following findings is considered as exclusion criteria: patients with serum creatinine $>1.4 \mathrm{mg} / \mathrm{dL}$, liver cell failure, neuropathy due to causes other than diabetes mellitus, CNS disorders.
$\mathrm{HbA}_{1 \mathrm{c}} \%$, serum creatinine, total cholesterol, triglycerides, HDL and LDL levels were measured. Serum $\beta$-endorphin was assayed by ELISA supplied by Elabscience (USA). The study design was case control study.

\section{Statistical analysis}

Data were tabulated, coded then analyzed using the computer program SPSS (Statistical package for social science) version 22.0 for Windows. Spearman correlation coefficient ${ }^{\circledR}$ test was used correlating different parameters. P value $<0.05$ was considered statistically significant in all analyses.

\section{Results}

The study included 57 diabetic subjects with diabetic peripheral neuropathy, 16 diabetic subjects without peripheral nerve dysfunction and 15 healthy control subjects.

The mean age of patients in the neuropathic group (53.91 \pm 5.53 years) was not significantly different from either the diabetic or control groups ( $50.37 \pm 7.56$ and $50.93 \pm 5.65$ years, respectively) $(p=0.053)$.

Male and female number and percentage ratio in the diabetic neuropathic group was $26(45.6 \%) / 31$ $(54.4 \%)$, in the diabetic non neuropathic group 8 $(50 \%) / 8(50 \%)$ and in the control group $8(53.3 \%) / 7$ $(46.7 \%)$ in male and feme respectively. The difference between all groups regarding gender was statistically insignificant $(p=0.850)$.

There was no significant difference between diabetic neuropathic, diabetic non neuropathic and control groups as regard body mass index $(p=0.489)$. BMl in the studied groups was [32.29 (28.1-38.3), 35.62 (27.675$-40.8)$ and $31.88(25.95-35.78) \mathrm{kg} / \mathrm{m}^{2}$, respectively].

Systolic BP wasn't significantly different $(p<0.084)$ between diabetic neuropathic, diabetic non neuropathic and control groups [130 (120-140), 120 (120-130) and $120(110-130) \mathrm{mm} \mathrm{Hg}$, respectively], also there was no significant difference regarding diastolic BP between the studied groups [80 (70-90), 70 (70-80) and $80(70-80) \mathrm{mm} \mathrm{Hg}$ respectively] $(p<0.336)$.

All subjects of the control group were normotensive, while most of the patients in the diabetic neuropathic and diabetic non neuropathic groups were hypertensive. There were 40 hypertensive patients $(70.2 \%)$ in the diabetic neuropathic group, and 9 patients $(56.2 \%)$ in the diabetic non neuropathic group. There was no significant difference between the two groups regarding the number of patients with hypertension ( $p=0.295)$. The duration of hypertension was significantly longer in the diabetic neuropathic versus diabetic non neuropathic groups [5 (0-15) and 0.5 $(0-4)$ years respectively] $(p<0.001)$. 
There was no significant difference between diabetic neuropathic and diabetic non neuropathic groups as regard the anti-hypertensive drugs $(p=0.028)$. In diabetic neuropathic and diabetic non neuropathic groups there were following number of patients receiving ACEls: 14 (24.6\%) and $6(37.5 \%)$ respectively, patients receiving BBs: 2 (3.5\%) and 1 (6.3\%) respectively, patients receiving CCBs: 3 (5.3\%) and 1 (6.3\%) respectively and patients receiving combinations of more than one antihypertensive drugs: 21 (36.9\%) and $1(6.3 \%)$ respectively.

Duration of diabetes was significantly longer in diabetic neuropathic group than in diabetic non neuropathic group [14 (8.5-20) versus 4 (1.125-9.5) years, respectively] ( $p<0.001$ ). Drug therapy for DM shows no significant difference among both diabetic neuropathic and diabetic non neuropathic groups ( $p=0.058$ ). Insulin was used by $19.3 \%$ (11 patients) of the diabetic neuropathic group versus 50\% (8 pa- tients) of the diabetic non neuropathic group, insulin + metformin was used by $54.4 \%$ (31 patients) of the diabetic neuropathic group versus $0 \%$ (0 patients) of the diabetic non neuropathic group, SU was used by $14 \%$ (11 patients) of the diabetic neuropathic group versus $25 \%$ (4 patients) of the diabetic non neuropathic group and $\mathrm{SU}+$ metformin was used by $14 \%$ (11 patients) of the diabetic neuropathic group versus $25 \%$ (4 patients) of the diabetic non neuropathic group.

Painful and painless neuropathy subgroups were matched for age $(53.709 \pm 6.414,54.153 \pm 4.378$ years respectively) ( $p=0.766$ ) and sex; in the painful neuropathy subgroup [11 males (35.5\%) and 20 females $(64.5 \%)$ ] and in the painless neuropathy subgroup [15 males (57.7\%) and 11 females (42.3\%)] $(p=0.094)$. BMl shows no significant difference between both subgroups $\left(34.69 \pm 7.03\right.$ and $31.79 \pm 6.15 \mathrm{~kg} / \mathrm{m}^{2}$ respectively) $(p=0.107)$.

Table 1. Demographic and clinical data of the studied groups

\begin{tabular}{|c|c|c|c|c|c|}
\hline \multirow[t]{2}{*}{ Data } & & \multicolumn{3}{|c|}{ Groups } & \multirow[t]{2}{*}{$\mathbf{p}$} \\
\hline & & $\begin{array}{c}\text { Diabetic neuropathy } \\
\text { present }\end{array}$ & $\begin{array}{l}\text { Diabetic neuropathy } \\
\text { absent }\end{array}$ & Control & \\
\hline \multirow[t]{2}{*}{ Age (years) } & Mean & 53.912 & 50.375 & 50.933 & 0.053 \\
\hline & $\pm S D$ & 5.536 & 7.562 & 5.65 & \\
\hline \multirow[t]{2}{*}{ Gender (N/\%) } & Male & $26(45.6 \%)$ & $8(50 \%)$ & $8(53.3 \%)$ & 0.850 \\
\hline & Female & $31(54.4 \%)$ & $8(50 \%)$ & $7(46.7 \%)$ & \\
\hline \multirow[t]{2}{*}{ BMI $\left[\mathrm{kg} / \mathrm{m}^{2}\right]$} & Median & 32.29 & 35.62 & 31.88 & 0.489 \\
\hline & Range & $28.1-38.3$ & $27.675-40.8$ & $25.95-35.78$ & \\
\hline \multirow[t]{2}{*}{$\mathrm{SBP}[\mathrm{mm} \mathrm{Hg}]$} & Median & 130 & 120 & 120 & 0.084 \\
\hline & Range & $120-140$ & $120-130$ & $110-130$ & \\
\hline \multirow[t]{2}{*}{$\mathrm{DBP}[\mathrm{mm} \mathrm{Hg}]$} & Median & 80 & 70 & 80 & 0.336 \\
\hline & Range & $70-90$ & $70-80$ & $70-80$ & \\
\hline \multirow[t]{2}{*}{ Hypertension (N/\%) } & Yes & $40(70.2 \%)$ & $9(56.2 \%)$ & & $<0.295$ \\
\hline & No & $17(29.8 \%)$ & $7(43.8 \%)$ & & \\
\hline Hypertension duration & Median & 5 & 0.5 & & $<0.001$ \\
\hline (years) & Range & $0-15$ & $0-4$ & & \\
\hline Hypertension treatment & ACEls & $14(24.6 \%)$ & $6(37.5 \%)$ & & 0.028 \\
\hline \multirow[t]{4}{*}{$(\mathrm{N} / \%)$} & BBs & $2(3.5 \%)$ & $1(6.3 \%)$ & & \\
\hline & CCBs & $3(5.3 \%)$ & $1(6.3 \%)$ & & \\
\hline & Combination & $21(36.9 \%)$ & $1(6.3 \%)$ & & \\
\hline & No & $17(29.8 \%)$ & $7(43.8 \%)$ & & \\
\hline \multirow[t]{2}{*}{ DM duration (years) } & Median & 14 & 4 & & $<0.001$ \\
\hline & Range & $8.5-20$ & $1.125-9.5$ & & \\
\hline \multirow[t]{4}{*}{ DM treatment $(\mathrm{N} / \%)$} & SU & $8(14 \%)$ & $4(25 \%)$ & & $<0.058$ \\
\hline & Insulin & $11(19.3 \%)$ & $8(50 \%)$ & & \\
\hline & Insulin + metformin & $31(54.4 \%)$ & $0(0 \%)$ & & \\
\hline & $\mathrm{SU}+$ metformin & $7(12.3 \%)$ & $4(25 \%)$ & & \\
\hline
\end{tabular}

P — probability; SD — standard deviation; BMI — body mass index; SBP — systolic blood pressure; DBP — diastolic blood pressure; ACEls — angiotensine converting enzyme inhibitors; BBs - beta-blockers; CCBs - calcium channel blockers; SU — sulphonylurea 
Table 2. Demographic and clinical data of the neuropathic group

\begin{tabular}{|c|c|c|c|c|}
\hline \multirow[t]{2}{*}{ Data } & & \multicolumn{2}{|c|}{ Subgroups } & \multirow[t]{2}{*}{$\mathbf{p}$} \\
\hline & & Painful neuropathy & Painless neuropathy & \\
\hline \multirow[t]{2}{*}{ Age (years) } & Mean & 53.709 & 54.153 & 0.766 \\
\hline & $\pm S D$ & 6.414 & 4.378 & \\
\hline \multirow[t]{2}{*}{ Gender (N/\%) } & Male & $11(35.5 \%)$ & $15(57.7 \%)$ & 0.094 \\
\hline & Female & $20(64.5 \%)$ & $11(42.3 \%)$ & \\
\hline \multirow[t]{2}{*}{ BMI $\left[\mathrm{kg} / \mathrm{m}^{2}\right]$} & Mean & 34.69 & 31.79 & 0.107 \\
\hline & $\pm S D$ & 7.03 & 6.15 & \\
\hline \multirow[t]{2}{*}{$\mathrm{SBP}[\mathrm{mm} \mathrm{Hg}]$} & Mean & 125.16 & 136.5 & 0.026 \\
\hline & $\pm S D$ & 15.464 & 19.815 & \\
\hline \multirow[t]{2}{*}{$\mathrm{DBP}[\mathrm{mm} \mathrm{Hg}]$} & Mean & 77.42 & 83.46 & 0.049 \\
\hline & $\pm S D$ & 10.318 & 11.981 & \\
\hline \multirow[t]{2}{*}{ Hypertension (N/\%) } & Yes & $21(67.7 \%)$ & $19(73.1 \%)$ & 0.774 \\
\hline & No & $10(32.3 \%)$ & $7(26.9 \%)$ & \\
\hline Hypertension duration & Median & 2 & 6 & 0.411 \\
\hline (years) & Range & $0-20$ & $0-21$ & \\
\hline Hypertension treatment & ACEls & $8(25.8 \%)$ & $6(23.1 \%)$ & 0.853 \\
\hline \multirow[t]{4}{*}{ (N/\%) } & BBs & $1(3.2 \%)$ & $1(3.8 \%)$ & \\
\hline & CCBs & $2(6.5 \%)$ & $1(3.8 \%)$ & \\
\hline & Combination & $10(32.3 \%)$ & $11(42.3 \%)$ & \\
\hline & No & $10(32.3 \%)$ & $7(26.9 \%)$ & \\
\hline \multirow[t]{2}{*}{ DM duration (years) } & Median & 15 & 11.5 & 0.146 \\
\hline & Range & $2-25$ & $3.5-25$ & \\
\hline \multirow[t]{4}{*}{ DM treatment (N/\%) } & SU & $3(9.7 \%)$ & $5(19.2 \%)$ & 0.443 \\
\hline & Insulin & 17 (54.8\%) & $14(53.5 \%)$ & \\
\hline & $\begin{array}{l}\text { Insulin + met- } \\
\text { formin }\end{array}$ & $8(25.8 \%)$ & $3(11.5 \%)$ & \\
\hline & $\mathrm{SU}+$ metformin & $3(9.7 \%)$ & $4(15.4 \%)$ & \\
\hline
\end{tabular}

P — probability; SD — standard deviation; BMI — body mass index; SBP — systolic blood pressure; DBP — diastolic blood pressure; ACEls — angiotensine converting enzyme inhibitors; BBs — beta-blockers; CCBs — calcium channel blockers; SU — sulphonylurea

Table 3. Clinical characteristics of pain in the painful neuropathy subgroup

\begin{tabular}{llc}
\hline Data & & (N/\%) \\
\hline Pain character (N/\%) & Pricking & $3(9.7 \%)$ \\
& Deep aching & $4(12.9 \%)$ \\
& Coldness & $2(6.5 \%)$ \\
& Tingling & $20(64.5 \%)$ \\
Pain severity (VAS) & Burning & $2(6.5 \%)$ \\
& Median & 7 \\
Pain therapy & Range & $5-8$ \\
& No treatment & $26(83.9 \%)$ \\
& Carbamazepine & $2(6.5 \%)$ \\
Other pains & Gabapentin & $2(6.5 \%)$ \\
& Pregabalin & $1(3.2 \%)$ \\
& No & $20(64.5 \%)$ \\
& OA & $8(25.8 \%)$ \\
\end{tabular}

VAS — visual analogue scale; OA — osteoarthritis 
Table 4. NDS of the studied groups

\begin{tabular}{|c|c|c|c|c|c|}
\hline \multirow[t]{2}{*}{ Data } & & \multicolumn{3}{|c|}{ Groups } & \multirow[t]{2}{*}{$\mathbf{p}$} \\
\hline & & $\begin{array}{c}\text { Diabetic neuropathy } \\
\text { present }\end{array}$ & $\begin{array}{l}\text { Diabetic neuropathy } \\
\text { absent }\end{array}$ & Control & \\
\hline \multirow[t]{2}{*}{ NDS: total score } & Median & 8 & 2 & 0 & $<0.001$ \\
\hline & Range & $6-10$ & $0-4$ & $0-0$ & \\
\hline \multirow[t]{2}{*}{ Vibration perception (RT) } & Median & 1 & 0 & 0 & $<0.001$ \\
\hline & Range & $1-1$ & $0-1$ & $0-0$ & \\
\hline \multirow[t]{2}{*}{ Vibration perception (LT) } & Median & 1 & 0 & 0 & $<0.001$ \\
\hline & Range & $1-1$ & $0-1$ & $0-0$ & \\
\hline Temperature perception & Median & 1 & 0 & 0 & $<0.001$ \\
\hline (RT) & Range & $0-1$ & $0-0$ & $0-0$ & \\
\hline Temperature perception & Median & 1 & 0 & 0 & $<0.001$ \\
\hline (LT) & Range & $0-1$ & $0-0$ & $0-0$ & \\
\hline \multirow[t]{2}{*}{ Pin prick (RT) } & Median & 1 & 0 & 0 & $<0.001$ \\
\hline & Range & $1-1$ & $0-0$ & $0-0$ & \\
\hline \multirow[t]{2}{*}{ Pin prick (LT) } & Median & 1 & 0 & 0 & $<0.001$ \\
\hline & Range & $1-1$ & $0-0$ & $0-0$ & \\
\hline \multirow[t]{2}{*}{ Achilles reflex (RT) } & Median & 2 & 0 & 0 & $<0.001$ \\
\hline & Range & $1-2$ & $0-0$ & $0-0$ & \\
\hline \multirow[t]{2}{*}{ Achilles reflex (LT) } & Median & 2 & 0 & 0 & $<0.001$ \\
\hline & Range & $2-2$ & $0-0$ & $0-0$ & \\
\hline
\end{tabular}

P - probability; NDS — neuropathy disability score; RT — right foot; LT — left foot

Table 5. NDS of the neuropathic group

\begin{tabular}{|c|c|c|c|c|}
\hline \multirow[t]{2}{*}{ Data } & & \multicolumn{2}{|c|}{ Subgroups } & \multirow[t]{2}{*}{ p } \\
\hline & & Painful neuropathy & Painless neuropathy & \\
\hline \multirow[t]{2}{*}{ NDS: total score } & Mean & 8.48 & 8.15 & 0.440 \\
\hline & $\pm S D$ & 1.568 & 1.617 & \\
\hline \multirow[t]{2}{*}{ Vibration perception (RT) } & Mean & 0.97 & 0.96 & 0.902 \\
\hline & $\pm S D$ & 0.180 & 0.196 & \\
\hline \multirow[t]{2}{*}{ Vibration perception (LT) } & Mean & 0.97 & 0.85 & 0.133 \\
\hline & $\pm S D$ & 0.180 & 0.368 & \\
\hline \multirow[t]{2}{*}{ Temperature perception (RT) } & Median & 1 & 1 & 0.312 \\
\hline & Range & $0-1$ & $0-1$ & \\
\hline \multirow[t]{2}{*}{ Temperature perception (LT) } & Median & 1 & 1 & 0.437 \\
\hline & Range & $0-1$ & $0-1$ & \\
\hline \multirow[t]{2}{*}{ Pin prick (RT) } & Median & 1 & 1 & 0.437 \\
\hline & Range & $0-1$ & $0-1$ & \\
\hline \multirow[t]{2}{*}{ Pin prick (LT) } & Median & 1 & 1 & 0.054 \\
\hline & Range & $0-1$ & $0-1$ & \\
\hline \multirow[t]{2}{*}{ Achilles reflex (RT) } & Mean & 1.71 & 1.81 & 0.475 \\
\hline & $\pm S D$ & 0.529 & 0.491 & \\
\hline \multirow[t]{2}{*}{ Achilles reflex (LT) } & Mean & 1.71 & 1.88 & 0.182 \\
\hline & $\pm S D$ & 0.588 & 0.326 & \\
\hline
\end{tabular}

P — probability; SD — standard deviation; NDS — neuropathy disability score; RT — right; foot; LT — left foot 
Table 6. Laboratory data of the studied groups

\begin{tabular}{|c|c|c|c|c|c|}
\hline \multirow[t]{2}{*}{ Data } & & \multicolumn{3}{|c|}{ Groups } & \multirow[t]{2}{*}{$\mathbf{p}$} \\
\hline & & $\begin{array}{l}\text { Diabetic neuro- } \\
\text { pathy present }\end{array}$ & $\begin{array}{l}\text { Diabetic neuro- } \\
\text { pathy absent }\end{array}$ & Control & \\
\hline \multirow[t]{2}{*}{$\beta$-endorphin [pg/mL] } & Median & 293.00 & 262.50 & 307.00 & 0.275 \\
\hline & Range & $26.00-1000.00$ & $14.00-700.00$ & $106.00-1000.00$ & \\
\hline \multirow[t]{2}{*}{$\mathrm{HbA}_{1 \mathrm{c}}(\%)$} & Mean & 8.700 & 8.206 & 5.043 & $<0.001$ \\
\hline & $\pm S D$ & 1.760 & 1.998 & 0.397 & \\
\hline \multirow[t]{2}{*}{ Serum creatinine $[\mathrm{mg} / \mathrm{dL}]$} & Mean & 0.815 & 0.762 & 0.713 & 0.103 \\
\hline & $\pm S D$ & 0.179 & 0.150 & 0.164 & \\
\hline \multirow[t]{2}{*}{ HDL-cholesterol [mg/dL] } & Median & 37.00 & 36.50 & 37.00 & 0.998 \\
\hline & Range & $25.00-76.00$ & $29.00-103.00$ & $32.00-52.00$ & \\
\hline \multirow[t]{2}{*}{ Total cholesterol [mg/dL] } & Median & 215.00 & 244.50 & 218.00 & 0.453 \\
\hline & Range & $118.00-491.00$ & $128.00-376.00$ & $172.00-359.00$ & \\
\hline \multirow[t]{2}{*}{ Triglycerides [mg/dL] } & Median & 155.00 & 132.50 & 130.00 & 0.769 \\
\hline & Range & $66.00-546.00$ & $73.00-232.00$ & $71.00-238.00$ & \\
\hline \multirow[t]{2}{*}{ LDL-cholesterol [mg/dL] } & Median & 150.00 & 165.00 & 149.60 & 0.341 \\
\hline & Range & $52.00-399.00$ & $87.40-307.00$ & $104.00-275.00$ & \\
\hline
\end{tabular}

$\mathrm{P}$ - probability; SD — standard deviation; $\mathrm{HbA}_{1 \mathrm{c}}$ - glycosylated hemoglobin; $\mathrm{HDL}$ - high-density lipoprotein; $\mathrm{LDL}$ - low-density lipoprotein

Table 7. Laboratory data of the neuropathic group

\begin{tabular}{|c|c|c|c|c|}
\hline \multirow[t]{2}{*}{ Data } & & \multicolumn{2}{|c|}{ Subgroups } & \multirow[t]{2}{*}{ p } \\
\hline & & Painful neuropathy & Painless neuropathy & \\
\hline \multirow[t]{2}{*}{$\beta$-endorphin $[\mathrm{pg} / \mathrm{mL}]$} & Median & 200 & 303 & 0.701 \\
\hline & Range & $26-1000$ & $39-800$ & \\
\hline \multirow[t]{2}{*}{$\mathrm{HbA}_{1 \mathrm{c}}(\%)$} & Mean & 9.061 & 8.269 & 0.091 \\
\hline & $\pm S D$ & 1.904 & 1.493 & \\
\hline \multirow[t]{2}{*}{ Serum creatinine $[\mathrm{mg} / \mathrm{dL}]$} & Mean & 0.787 & 0.850 & 0.196 \\
\hline & $\pm S D$ & 0.164 & 0.192 & \\
\hline \multirow[t]{2}{*}{ HDL-cholesterol [mg/dL] } & Mean & 40.548 & 38.461 & 0.466 \\
\hline & $\pm S D$ & 10.859 & 10.485 & \\
\hline \multirow[t]{2}{*}{ Total cholesterol [mg/dL] } & Mean & 240.483 & 214.080 & 0.196 \\
\hline & $\pm S D$ & 74.955 & 76.652 & \\
\hline \multirow[t]{2}{*}{ Triglycerides [mg/dL] } & Median & 154 & 158 & 0.749 \\
\hline & Range & $69-277$ & $66-546$ & \\
\hline \multirow[t]{2}{*}{ LDL-cholesterol [mg/dL] } & Median & 174 & 129.7 & 0.062 \\
\hline & Range & $72-310$ & $52-399$ & \\
\hline
\end{tabular}

$\mathrm{P}$ - probability; SD — standard deviation; $\mathrm{HbA}_{1 \mathrm{c}}$ - glycosylated hemoglobin; $\mathrm{HDL}$ - high-density lipoprotein; $\mathrm{LDL}$ - low-density lipoprotein

Table 8. Serum $\beta$-endorphin in patients using treatment for neuropathic pain versus non-users

\begin{tabular}{|c|c|c|c|c|c|}
\hline \multirow[t]{3}{*}{ Data } & \multicolumn{4}{|c|}{ Pain treatment } & \multirow[t]{3}{*}{$\mathbf{p}$} \\
\hline & \multicolumn{2}{|c|}{ Non users } & \multicolumn{2}{|c|}{ Users } & \\
\hline & Median & Range & Median & Range & \\
\hline$\beta$-endorphin [pg/mL] & 307 & $14-1000$ & 104 & $53-451$ & 0.117 \\
\hline
\end{tabular}




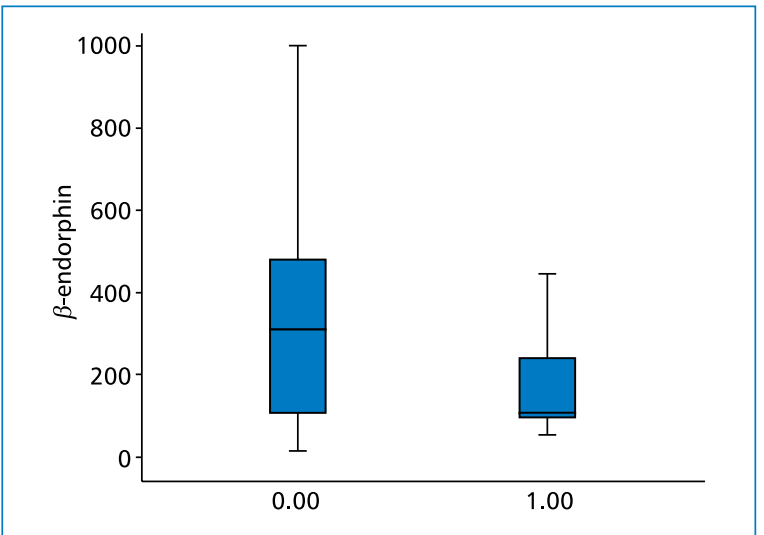

Figure 1. 0.00: patients not using treatment for neuropathic pain; 1.00: patients using treatment for neuropathic pain

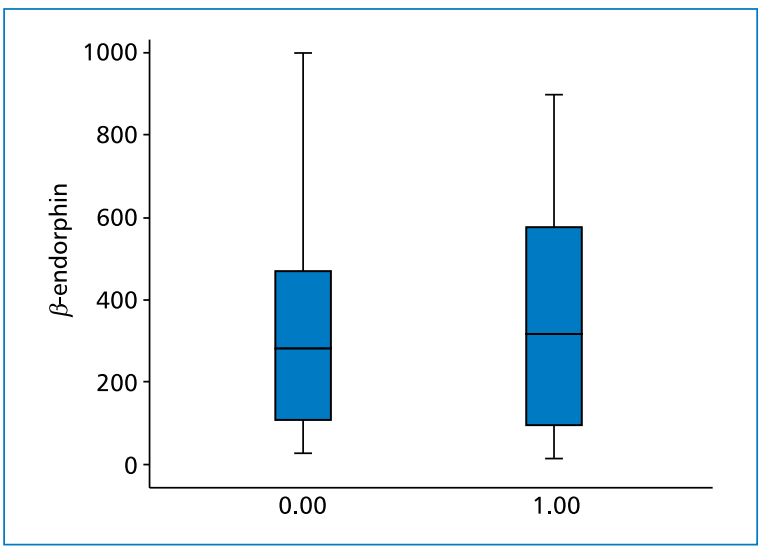

Figure 2. 0.00: patients without associated pain; 1.00: patients with associated pain

Table 9. Serum $\beta$-endorphin in patients presenting with pain versus those without pain

\begin{tabular}{|c|c|c|c|c|c|}
\hline \multirow[t]{3}{*}{ Data } & \multicolumn{4}{|c|}{ Associated pain } & \multirow[t]{3}{*}{$p$} \\
\hline & \multicolumn{2}{|c|}{ Absent } & \multicolumn{2}{|c|}{ Present } & \\
\hline & Median & Range & Median & Range & \\
\hline$\beta$-endorphin [pg/mL] & 283 & $26-1000$ & 315 & $14-950$ & 0.974 \\
\hline
\end{tabular}

Table 10. Serum $\beta$-endorphin in patients having different characters of pain

\begin{tabular}{llcccccc}
\hline \multirow{2}{*}{ Data } & \multicolumn{4}{c}{ Character of pain } & p \\
\cline { 3 - 7 } & & Pricking & Deep aching & Coldness & Numbness & Burning & \\
\hline$\beta$-endorphin $[\mathrm{pg} / \mathrm{ml}]$ & Median & 104 & 142 & 60.5 & 330.5 & 436 & 0.176 \\
& Range & $49-680$ & $51.7-399$ & $26-95$ & $53.1-1000$ & $397-475$ & \\
\hline
\end{tabular}

SBP and DBP were significantly lower in the painful than painless neuropathy subgroup, $(125.16 \pm 15.46$ and $136.5 \pm 19.81 \mathrm{~mm} \mathrm{Hg})(p=0.026)$ and $(77.42$ \pm 10.31 and $83.46 \pm 11.98 \mathrm{~mm} \mathrm{Hg})(p=0.049)$ respectively. History of hypertension (HTN) wasn't significantly different between both subgroups, in painful neuropathy subgroup; 21 patients (67.7\%) were hypertensive while in painless neuropathy subgroup; 19 patients $(73.1 \%)$ were hypertensive $(p=0.774)$. As regard duration of HTN, there was no significant difference between painful and painless neuropathy subgroups [2 (0-20) and $6(0-21)$ years respectively] $(p=0.411)$. Treatment of HTN also shows no significant difference; in painful neuropathy subgroup ACEls were used in 8 patients $(25.8 \%)$, BBs in 1 patient $(3.2 \%)$, CCBs in 2 patients (6.5\%) and combinations of more than one antihypertensive in 10 patients (32.3\%) while in painless neuropathy subgroup ACEls were used in 6 patients $(23.1 \%)$, BBs in 1 patient $(3.8 \%)$, CCBs in 1 patient (3.8\%) and combinations of more than one antihypertensive in 11 patients $(42.3 \%)(p=0.853)$.
DM duration shows no significant difference between painful and painless neuropathy subgroups [15 (2-25) and $11.5(3.5-25)$ years respectively] $(p=0.146)$. There was no significant difference regarding DM treatment, in painful neuropathy subgroup; SU were used in $9.7 \%$ (3 patients), insulin was used in $54.8 \%$ (17 patients), insulin + metformin were used in $25.8 \%$ (8 patients) and SU + metformin were used in $9.7 \%$ (3 patients) while in painless neuropathy subgroup; SU was used in $19.2 \%$ (5 patients), insulin was used in $53.5 \%$ (14 patients), insulin + metformin were used in $11.5 \%$ ( 3 patients) and SU + metformin were used in $15.4 \%$ (4 patients) ( $p=0.443$ ).

The most prevalent pain character was tingling (64.5\%), other were deep aching pain (12.9\%), pricking pain $(9.7 \%)$, coldness $(6.5 \%)$ and burning (6.5\%). In addition, pain severity on VAS ranges from 5 to $8.83 .9 \%$ of neuropathic patient were not using pain-controlling medications and $64.5 \%$. had no other causes of chronic pain.

The neuropathy disability score (NDS) was used to diagnose peripheral neuropathy, total score $\geq 6$ 
Table 11. Serum $\beta$-endorphin $[\mathrm{pg} / \mathrm{mL}]$ in different modalities of nerve disability

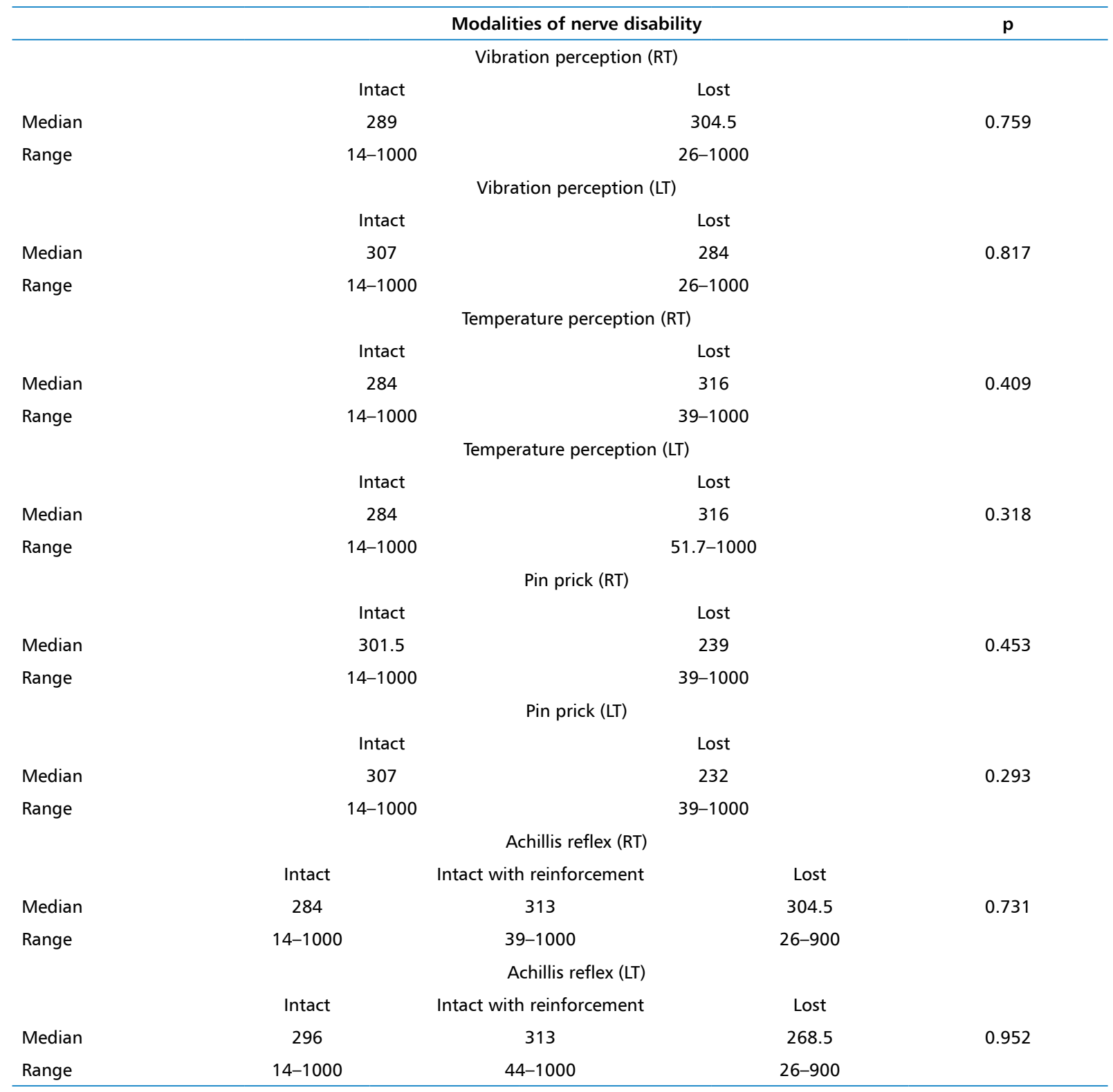

p - probability; test used - Mann-Whitney U

was diagnostic for peripheral neuropathy. Total score was significantly higher in diabetic neuropathic versus diabetic non neuropathic and control groups; [8 (6-10) versus $2(0-4)$ and $0(0-0 \%)$ respectively] $(p<0.001)$.

Vibration perception threshold score was significantly higher in diabetic neuropathic versus diabetic non neuropathic and control groups; at the right foot [1 (1-1) versus $0(0-1)$ and $0(0-0)$ respectively] $(p<0.001)$, at the left foot it was the same respectively $(p<0.001)$. Temperature perception score also was significantly higher in diabetic neuropathic versus diabetic non neuropathic and control groups; at the right foot [1 (0-1) versus $0(0-0)$ and $0(0-0)$ respectively] $(p<0.001)$, at the left foot it was the same respectively $(p<0.001)$.
Pin prick sensation score was significantly higher in diabetic neuropathic versus diabetic non neuropathic and control groups; at the right foot, [1 (1-1) versus 0 $(0-0)$ and $0(0-0)$ respectively] $(p<0.001)$, at the left foot it was the same respectively $(p<0.001)$. Achillis reflex score was significantly higher in diabetic neuropathic versus diabetic non neuropathic and control groups; at the right foot, [2 (1-2) versus $0(0-0)$ and 0 $(0-0)$ respectively] $(p<0.001)$, at the left foot $[2(2-2)$ versus $0(0-0)$ and $0(0-0)$ respectively] $(p<0.001)$.

In painful and painless neuropathy subgroups, NDS: total score showed no significant difference [8.48 \pm 1.56 and $8.15 \pm 1.61$ respectively] $(p=0.440)$. With regard to vibration perception threshold score 


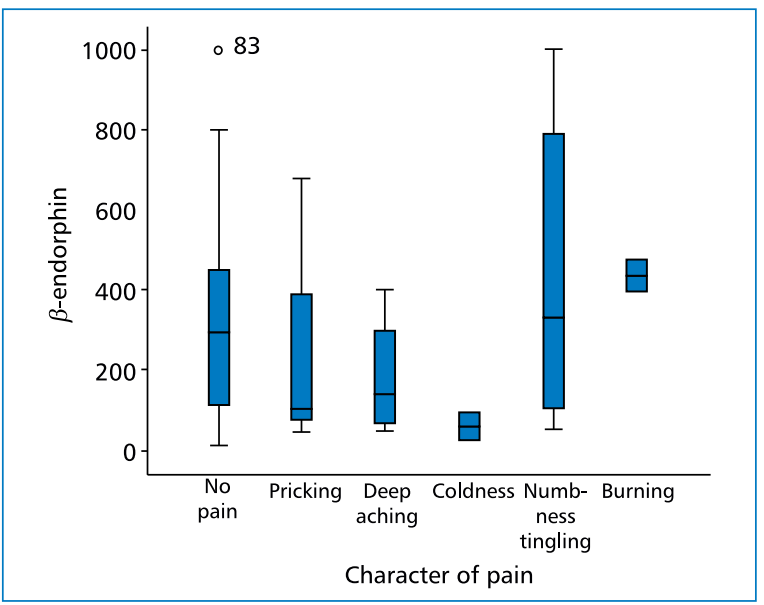

Figure 3. Serum levels of $\beta$-endorphins according to type of pain

there was no significant difference between painful and painless neuropathy subgroups; at the right foot it was $[0.97 \pm 0.18$ and $0.96 \pm 0.196$ respectively] $(p=0.902)$, at the left foot it was $[0.97 \pm 0.18$ and $0.85 \pm 0.368$ respectively] $(p=0.133)$.

No significant difference between painful and painless neuropathy subgroups regarding temperature perception score; at the right foot [1 (0-1) and $1(0-1)$ respectively] ( $p=0.312)$, at the left foot [1 $(0-1)$ and $1(0-1)$ respectively] $(p=0.437)$. Pin prick sensation score showed no significant difference between painful and painless neuropathy subgroups; at the right foot [1 $(0-1)$ and $1(0-1)$ respectively] $(p=0.437)$, at the left foot [1 (0-1) and $1(0-1)$ respectively] $(p=0.054)$. Achillis reflex score showed no significant difference between painful and painless neuropathy subgroups; at the right foot $[1.71 \pm 0.529$ and $1.81 \pm 0.491$ respectively] $(p=0.475)$, at the left foot $[1.71 \pm 0.588$ and $1.88 \pm 0.326$ respectively] $(p=0.182)$.

$\mathrm{HbA}_{1 \mathrm{c}}$ levels were higher in the diabetic neuropathic and diabetic non neuropathic groups in comparison to the control group $(8.700 \pm 1.760,8.206 \pm 1.998$ and $5.043 \pm 0.397 \%$ respectively) $(p<0.001)$. Serum creatinine levels showed no significant difference among diabetic neuropathic, diabetic non neuropathic and control groups $(0.815 \pm 0.179,0.762 \pm 0.150,0.713$ $\pm 0.164 \mathrm{mg} / \mathrm{dL}$ respectively) $(\mathrm{p}=0.103)$.

Regarding serum HDL-cholesterol and serum LDL-cholesterol levels there was no significant difference between diabetic neuropathic, diabetic non neuropathic and control groups [37 (25-76), 35.5 (29-103) and $37(32-52) \mathrm{mg} / \mathrm{dL}$ respectively] $(\mathrm{p}=0.998)$ and [150 (52-399), 165 (87.40-307) and 149.60 (104-275) mg/dL respectively] ( $p=0.341)$.
Table 12. Correlation between serum $\beta$-endorphin and other variables

\begin{tabular}{lcc}
\hline Data & \multicolumn{2}{c}{$\beta$-endorphin } \\
\cline { 2 - 3 } & $\mathbf{r}$ & $\mathbf{P}$ \\
\hline Severity of pain & -0.097 & 0.371 \\
NDS: total score & 0.027 & 0.803 \\
Age & -0.210 & 0.049 \\
BMI & 0.027 & 0.801 \\
DM duration & 0.056 & 0.607 \\
HbA $_{1 c} \%$ & -0.204 & 0.048 \\
Serum creatinine & 0.191 & 0.074 \\
\hline
\end{tabular}

NDS - neuropathy disability score; $\mathrm{BMI}$ - body mass index; $\mathrm{HbA}_{1 \mathrm{c}}$ - glycosylated hemoglobin

There was no significant difference between diabetic neuropathic, diabetic non neuropathic and control regarding serum total cholesterol and triglycerides [215 (118-491), 244.5 (128-376) and 218 (172-359) $\mathrm{mg} / \mathrm{dL})$ respectively] $(\mathrm{p}=0.453)$ and $[155(66-546)$, $132.5(73-232)$ and $130(71-238) \mathrm{mg} / \mathrm{dL}$ respectively] $(p=0.769)$.

Serum $\beta$-endorphin levels showed no significant difference between diabetic neuropathic, diabetic non neuropathic and control groups [293 (26-1000), 262.5 (14-700) and 307 (106-1000) pg/mL respectively] $(p=0.275)$

$\mathrm{HbA}_{1 \mathrm{c}} \%$ showed no significant difference between painful and painless neuropathy subgroups (9.061 \pm 1.904 and $8.269 \pm 1.493 \%$ respectively) $(p=0.091)$. Painful and painless neuropathy subgroups showed no significant difference regarding serum creatinine $(0.787 \pm 0.164$ and $0.850 \pm 0.192 \mathrm{mg} / \mathrm{dL}$ respectively) $(p=0.196)$.

Serum HDL-cholesterol and LDL-cholesterol showed no significant difference between both painful and painless neuropathy subgroups; serum HDL-cholesterol was ( $40.548 \pm 10.859$ and $38.461 \pm 10.485 \mathrm{mg} / \mathrm{dL}$ respectively) $(\mathrm{p}=0.466)$ and serum LDL-cholesterol was [174 (72-310) and 129.7 (52-399) $\mathrm{mg} / \mathrm{dL}$ respectively] $(p=0.062)$.

In addition, there was no significant difference between both painful and painless neuropathy subgroups regarding serum total cholesterol and triglycerides $(240.483 \pm 75$ and $214.080 \pm 76.652 \mathrm{mg} / \mathrm{dL}$ respectively) ( $p=0.196)$ and $[154(69-277)$ and 158 (66-546) $\mathrm{mg} / \mathrm{dL}$ respectively] ( $p=0.749$ ) respectively.

Serum $\beta$-endorphin shows no significant difference between painful and painless neuropathy subgroups [200 (26-1000) and 303 (39-800) pg/mL respectively] $(p=0.701)$.

Regarding serum $\beta$-endorphin there was no significant difference between patients who used pain con- 
trolling medications and those who did not use them [104 (53-451) and 307 (14-100) pg/mL respectively] $(p=0.117)$. There was no significant difference between diabetic neuropathic patients with or without associated pain regarding serum $\beta$-endorphin levels [315 (14-950) and $283(26-1000) \mathrm{pg} / \mathrm{mL}](\mathrm{p}=0.974)$. There were no significant difference in serum $\beta$-endorphin levels between patients with different characters of pain $(p=0.176)$.

There was no significant difference between lost and intact different modalities of nerve disability in either right and left foot as regard serum $\beta$-endorphin levels. Difference between intact and lost vibration perception as regard $B$ endorphin in the right foot [289 (14-1000) \& $304.5(26-1000)$ respectively] $(p=0.759)$, and in the left foot $[307$ (14-1000) \& 284 (26-1000) respectively] $(p=0.817)$. Difference between intact and lost temperature perception in the right foot [284 (14-1000) \& 316 (39-1000) respectively] $(p=0.409)$, and in the left foot [284 (14-1000) \& 316 (51.7-1000) respectively] ( $p=0.318)$. Difference between intact and lost pin prick sensation in the right foot [301.5 $(14-1000) \& 239(39-1000)$ respectively] $(p=0.453)$ and in the left foot [307 (14-1000) \& 232 (39-1000) respectively] $(p=0.293)$. Difference between intact achillis reflex, intact achillis reflex with reinforcement and lost achillis reflex in the right foot [284 (14-1000), 313 (39-1000), $304.5(26-900)$ respectively] $(p=0.731)$, andin the left foot [296 (14-1000), 313 (44-1000) and $268.5(26-900)$ respectively] ( $p=0.952)$.

Serum $\beta$-endorphin levels showed negative correlation with age $(r=-0.210, p=0.049)$ and $\mathrm{HA}_{1 \mathrm{c}} \%$ $(r=-0.204, p=0.048)$. However, it did not correlate with pain severity $(r=-0.097, p=0.371)$, NDS: total score $(r=0.027, p=0.803), \mathrm{BMI}(r=0.027, p=0.801)$, s. creatinine $(r=0.191, p=0.074)$ or DM duration $(r=0.056, p=0.607)$

\section{Discussion}

The age of our patients ranged from 40-65 years and there was no significant difference regarding age between various groups $(p=0.053)$. Painful and painless neuropathy subgroups were also matched for age $(p=0.766)$. Age is a well defined risk factors for diabetic peripheral neuropathy in observational as well as intervention studies $[11,12]$. In this work, we couldn't study the effect of age as we selected all our patients from the age of 40 to 65 and we tried to make all groups matched for age.

In our study males constituted $47.7 \%$ of the total number of subjects included in the study and females constituted $52.3 \%$ and there was no significant difference between the groups regarding gender $(p=0.850)$. Painful and painless neuropathy subgroups were matched for sex $(p=0.094)$. Although female gender was associated with painful DPN in some studies [13-15], in our study we have nearly equal distribution between males and females so the effect of gender couldn't be assessed.

DM duration was longer in the neuropathic group [14 (8.2-20) years] versus the diabetic group [4 (1.125$-9.5)$ years] ( $p<0.001)$. Tesfaye et al. (2005) and Shaw et al. (2003) reported that diabetes duration is a well known risk factor for diabetic peripheral neuropathy. However, peripheral neuropathy was documented in cases with prediabetes. The pathogenic mechanisms of prediabetic neuropathy are multiple and not completely understood. Chronic hyperglycemia, dyslipidemia, microangiopathy, and factors of the metabolic syndrome have been implicated [16]. In painful and painless neuropathy subgroups, DM duration showed no significant difference [15 (2-25) and 11.5 (3.5-25) years respectively] ( $p=0.146)$. Diabetes duration was found to be a correlate or predictor for painful DPN in some studies $[15,17]$ but not in others $[18,19]$.

There was no significant difference between various groups regarding BMI $(p=0.489)$. Most of the subjects were obese with $\mathrm{BMI}>30 \mathrm{~kg} / \mathrm{m}^{2}$ which constitute $68.42 \%$ of the neuropathic group, $75 \%$ of the diabetic group and $60 \%$ of the control group. In painful and painless neuropathy subgroups, BMI was (34.7 \pm $\pm 7 \mathrm{~kg} / \mathrm{m}^{2}$ and $31.8 \pm 6 \mathrm{~kg} / \mathrm{m}^{2}$ respectively) $(p=0.107)$. Ziegler et al. and Jambart et al., reported that obesity $\left(B M I \geq 30 \mathrm{~kg} / \mathrm{m}^{2}\right)$ was related to painful DPN and have been suggested as a risk marker [15, 18]. Also Maury and Brichard, suggested that obesity represents a risk factor for neuropathic pain as a component of the metabolic syndrome and as a cause of many processes in the pathogenesis of DPN [20].

Hypertension was associated with diabetes in $70.2 \%$ of the neuropathic group and $56.2 \%$ of the diabetics without neuropathy $(p=0.295)$. Hypertension was reported as risk factors for diabetic peripheral neuropathy [11]. In our study, regarding to painful and painless neuropathy subgroups: there were $67.7 \%$ and $73.1 \%$ patients with hypertension respectively $(p=0.774)$, beside that SBP and DBP were significantly lower in painful neuropathy group when compared to painless neuropathy group $(p=0.026)(p=0.049)$. However, hypertension was found as a risk factor for painful DPN in some studies $[15,17]$.

In our study, the character of pain in the painful neuropathy subgroup was mainly in the form of tingling (64.5\%), deep aching (12.9\%), pricking in $9.7 \%$ coldness in $6.5 \%$ and burning in $6.5 \%$. Spallone et al., studied 59 patients with painful DPN and found paresthesia and burning as the most frequent sensory descriptors followed by paroxysmal pain (electric shock 
and stabbing), evoked pain (by brushing, pressure and cold), and deep pain (squeezing and pressure) [21]. Baron et al., found that the association between burning, prickling, and numbness was the most common pain sensory profile (26\%), followed by the pattern of pain attacks (16\%), burning with both prickling and allodynia without numbness (13\%), and allodynia with hyperalgesia (9\%) [22].

In our study, NDS was significantly higher in neuropathic group in comparison to diabetic and control groups ( $p<0.001$ ), however, there were no significant differences between painful and painless neuropathy subgroups regarding all items of NDS. Veves et al., suggested that neuropathic pain could be present at any stage of DPN, from subclinical to very late neuropathy [23]. Veves et al., also found that the score of sensory deficits was higher in patients with painful than in those with painless DPN [24]. Another study found that painful DPN patients had worse sensorimotor deficits in comparison to patients with nonpainful DPN [25]. While Boulton et al., reported that painful symptoms improve with the worsening of the sensory loss [26]. Pain in painful DPN would appear to be mainly associated with the impairment of small afferent fibers, however it couldn't be confirmed that there is no small fiber impairment in the absence of pain [27]. Krämer et al., found that those with painful DPN showed no significant difference in vibration perception thresholds and thermal thresholds in comparison to those with painless DPN [28]. Vrethem et al., detected that tactile sensitivity but not thermal sensitivity were more compromised in diabetic patients with painful compared with those with painless DPN [29].

In our study, we found no significant difference regarding serum $\beta$-endorphin levels neither between diabetic neuropathic, diabetic non neuropathic and control groups $(p=0.275)$, or between the painful neuropathy subgroup and painless neuropathy subgroup $(p=0.701)$.

In the literature, few studies were found to study $\beta$-endorphin level in patients with diabetic neuropathy. Cakir et al., conducted a study on 7 diabetic patients with painful DN, 7 diabetic patients without DN, and 7 healthy control subjects to investigate the efficacy of L-carnitine (LC) in the treatment of painful diabetic neuropathy (DN) and its effects on levels of the endogenous peptide $\beta$-endorphin. They found no difference in basal $\beta$-endorphin levels between diabetic patients with painful DN and diabetic patients without neuropathy and they also spotted that basal $\beta$ endorphin levels were significantly lower in the diabetic patients in comparison to the control group [30]. Tsigos et al., reported that $\beta$-endorphin concentrations are reduced in the CSF of patients with sensory diabetic polyneuropathy. However the difference wasn't statistically significant between those patients who have painful and those who have painless neuropathy [31]. In our study we found no significant correlation between $\beta$-endorphin concentrations and severity of pain $(r=-0.097, p=0.371)$. However, $\beta$-endorphin levels were significantly negatively correlated with age $(r=-0.210, p=0.049)$ and $\mathrm{HA}_{1 \mathrm{c}} \%(r=-0.204, \mathrm{p}=0.048)$. The effect of age on $\beta$-endorphin level was previously suggested by Goodwin et al., who described that $\beta$-endorphin levels were significantly negatively correlated with age [32].

Serum $\beta$-endorphin levels showed no significant difference in our patients how used pain controlling medications versus those who did not use $(p=0.117)$. Bäckryd et al., found no significant correlation between CSF $\beta$ endorphin level and use of pain medications in patients with chronic neuropathic pain [9]. However, Tramadol which is a drug with mild opiate properties had evidence from randomized controlled trials showing that tramadol is an effective treatment for neuropathic pain [33].

Serum $\beta$-endorphin levels in patients with associated pain versus those without showed no significant difference $(p=0.974)$. Serum $\beta$-endorphin in patients with different characters of pain showed no significant difference $(p=0.176)$. There was no significant difference between lost and intact different modalities of nerve disability (vibration perception, pain sensation and temperature perception) in either right or left foot as regard serum $\beta$-endorphin levels. To our knowledge, no previous studies addressed these topics.

The complex nature of pain pathway involving many pro and anti-nociceptive mediators could explain why $\beta$-endorphin concentration doesn't show significant difference in our study. Another explanation can be that central changes may be not reflected peripherally also it may be wise to measure both pro and anti-nociceptive markers at the same time. This explanation is compatible with the study of Veening et al., who observed that peripheral administration of $\beta$-endorphin does not necessarily induce the same effects as intra-cerebroventricular administration; this suggests the existence of two functionally different $\beta$-endorphin systems, one for the central effects and one for the peripheral effects. They concluded that CSF-levels of $\beta$-endorphin are not a reflection of its peripheral levels [34]. Also Bäckryd et al., reported that there are probably two functionally different $\beta$-endorphin systems: one peripheral (release of $\beta$-endorphin by the pituitary into the systemic circulation) and one central (synthesis in hypothalamic pro-opio-melanocortin (POMC) neurons) [9], with an intact blood-brain barrier (BBB) hinders free exchange of $\beta$-endorphin between plasma and CSF [35]. 
In addition, short half-life of $\beta$-endorphin was reported in some studies, which means that spikes for its serum concentration may be missed during the assay. Foley et al., reported that the mean terminal half-life after intravenous administration of 5 or $10 \mathrm{mg}$ of hu$\operatorname{man} \beta$-endorphin was $37 \mathrm{~min}$ [36]. Veening et al., also reported that half-life values of $\beta$-endorphin vary from 20 to $50 \mathrm{~min}$ in the human circulation [34]. Butelman et al., reported that full-length $\beta$-endorphin was detectable in all subjects up to 5 minutes after intravenous administration [37]. Moreover, Bruehl et al., reported that chronic pain may initially be associated with upregulation of endogenous opioid analgesic systems, which then may become dysfunctional over time [38].

$\mathrm{HbA}_{1 \mathrm{c}} \%$ was significantly higher in diabetic neuropathic and diabetic non neuropathic groups in comparison to control group (8.7 $\pm 1.8 \%, 8.2 \pm 2 \%$ and $5 \pm 0.4 \%$ respectively) ( $\leq 0.001$ ), but there was no significant difference between painful and painless neuropathy subgroups $(9.061 \pm 1.904 \%$ and $8.269 \pm$ $\pm 1.493 \%$ respectively) ( $p=0.091)$. These finding is in accordance with Tesfaye et al., who reported that poor glycemic control is a risk factor for diabetic peripheral neuropathy [11]. However, Jin et al., reported that the best-known pathogenic factor of DPN is hyperglycemia-induced oxidative stress, and these oxidative stresses are worsened by acute glucose fluctuations [39]. Xu et al., found that glycemic variability assessed by continuous glucose monitoring was significantly independent risk factor for DPN in type 2 diabetes with well-controlled $\mathrm{HbA}_{1 \mathrm{c}} \%\left(\mathrm{HbA}_{1 \mathrm{c}}<7.0 \%\right)$ [40]. Oyibo et al., found that patients with painful neuropathy had greater glycemic excursions and possibly poorer diabetes control, compared with patients with painless neuropathy [41].

In our study lipid profile evaluation showed no significant difference between various groups. Padilla et al, reported that dyslipidemia is linked to diabetic neuropathy, and several underlying mechanisms have been identified, free fatty acids have been shown to directly cause injury to Schwann cells in vitro, and also have systemic effects such as promoting inflammatory cytokine release from adipocytes and macrophages [42]. Kassem et al., found that hypertriglyceridemia affects conduction parameters in peripheral nerves in a trend suggestive of early peripheral neuropathy [43].

In Our study, we found no significant differences regarding serum $\beta$-endorphin levels neither between diabetic neuropathic, diabetic non neuropathic and control groups, or between painful and painless neuropathy subgroups. There were no significant differences regarding serum $\beta$-endorphin levels between patients with different characters of pain and there were no correlations to pain severity. There were no significant differences between lost and intact different modalities of nerve disability with regard to serum $\beta$-endorphin levels.

\section{REFERENCES}

1. Pasnoor M, Dimachkie MM, Kluding P, et al. Diabetic neuropathy part 1: overview and symmetric phenotypes. Neurol Clin. 2013; 31(2): 425-445, doi: 10.1016/j.ncl.2013.02.004, indexed in Pubmed: 23642717.

2. Haanpää ML, Gourlay GK, Kent JL, et al. Treatment considerations for patients with neuropathic pain and other medical comorbidities. Mayo Clin Proc. 2010; 85(3 Suppl): S15-S25, doi: 10.4065/ /mcp.2009.0645, indexed in Pubmed: 20194144.

3. Selvarajah D, Wilkinson ID, Davies J, et al. Central nervous system involvement in diabetic neuropathy. Curr Diab Rep. 2011; 11(4): 310-322, doi: 10.1007/s11892-011-0205-z, indexed in Pubmed: 21667355.

4. Selvarajah D, Wilkinson ID, Emery CJ, et al. Early involvement of the spinal cord in diabetic peripheral neuropathy. Diabetes Care. 2006; 29(12): 2664-2669, doi: 10.2337/dc06-0650, indexed in Pubmed: 17130202.

5. Koob GF. Drugs of abuse: anatomy, pharmacology and function of reward pathways. Trends Pharmacol Sci. 1992; 13(5): 177-184, doi: 10.1016/0165-6147(92)90060-j, indexed in Pubmed: 1604710

6. Sprouse-Blum AS, Smith G, Sugai D, et al. Understanding endorphins and their importance in pain management. Hawaii Med J. 2010; 69(3): 70-71, indexed in Pubmed: 20397507.

7. Brunton L. Goodman and Gilman's The Pharmacological Basis of Therapeutics. McGraw-Hill, New York 2006: 547-559.

8. Miller R. Miller's Anesthesia. 6th ed. Elsevier, Pennsylvania 2005: 382-386.

9. Bäckryd E, Ghafouri B, Larsson B, et al. Do low levels of beta-endorphin in the cerebrospinal fluid indicate defective top-down inhibition in patients with chronic neuropathic pain? A crosssectional, comparative study. Pain Med. 2014; 15(1): 111-119, doi: 10.1111/pme.12248, indexed in Pubmed: 24118997.

10. American Diabetes Association. Standards of Medical Care in Diabetes - 2015. Diabetes Care. 2015; 38(1): S1-S93.

11. Tesfaye S, Chaturvedi N, Eaton SEM, et al. EURODIAB Prospective Complications Study Group. Vascular risk factors and diabetic neuropathy. N Engl J Med. 2005; 352(4): 341-350, doi: 10.1056/ /NEJMoa032782, indexed in Pubmed: 15673800.

12. Shaw JE, Zimmet PZ, Gries FA, Ziegler D. Epidemiology of diabetic neuropathy. In: Shaw JE, Zimmet PZ, Gries FA, Ziegler D. ed. Textbook of diabetic neuropathy. Thieme, Stuttgard 2003: 64-82.

13. Abbott CA, Malik RA, van Ross ERE, et al. Prevalence and characteristics of painful diabetic neuropathy in a large communitybased diabetic population in the U.K. Diabetes Care. 2011; 34(10): 2220-2224, doi: 10.2337/dc11-1108, indexed in Pubmed: 21852677.

14. Halawa MR, Karawagh A, Zeidan A, et al. Prevalence of painful diabetic peripheral neuropathy among patients suffering from diabetes mellitus in Saudi Arabia. Curr Med Res Opin. 2010; 26(2): 337-343, doi: 10.1185/03007990903471940, indexed in Pubmed: 19968592.

15. Jambart S, Ammache Z, Haddad F, et al. Prevalence of painful diabetic peripheral neuropathy among patients with diabetes mellitus in the Middle East region. J Int Med Res. 2011; 39(2): 366-377, doi: 10.1177/147323001103900204, indexed in Pubmed: 21672340.

16. Papanas N, Ziegler D. Prediabetic neuropathy: does it exist? Curr Diab Rep. 2012; 12(4): 376-383, doi: 10.1007/s11892-012-0278-3, indexed in Pubmed: 22562652. 
17. Van Acker K, Bouhassira D, De Bacquer D, et al. Prevalence and impact on quality of life of peripheral neuropathy with or without neuropathic pain in type 1 and type 2 diabetic patients attending hospital outpatients clinics. Diabetes Metab. 2009; 35(3): 206-213, doi: 10.1016/j.diabet.2008.11.004, indexed in Pubmed: 19297223

18. Ziegler D, Rathmann W, Dickhaus T, et al. KORA Study Group. Neuropathic pain in diabetes, prediabetes and normal glucose tolerance: the MONICA/KORA Augsburg Surveys S2 and S3. Pain Med. 2009; 10(2): 393-400, doi: 10.1111/j.1526-4637.2008.00555.x, indexed in Pubmed: 19207236.

19. Spallone V, Morganti R, D'Amato C, et al. Clinical correlates of painful diabetic neuropathy and relationship of neuropathic pain with sensorimotor and autonomic nerve function. Eur J Pain. 2011; 15(2): 153-160, doi: 10.1016/j.ejpain.2010.06.011, indexed in Pubmed: 20619708.

20. Maury E, Brichard SM. Adipokine dysregulation, adipose tissue inflammation and metabolic syndrome. Mol Cell Endocrinol. 2010; 314(1): 1-16, doi: 10.1016/j.mce.2009.07.031, indexed in Pubmed: 19682539.

21. Spallone V, Morganti R, Greco C, et al. Sensory profiles of neuropathic pain in painful diabetic polyneuropathy. Paper presented at the Diabetologia. SPRINGER. 2011; 54: 460-471.

22. Baron R, Tölle TR, Gockel U, et al. A cross-sectional cohort survey in 2100 patients with painful diabetic neuropathy and postherpetic neuralgia: Differences in demographic data and sensory symptoms. Pain. 2009; 146(1-2): 34-40, doi: 10.1016/j. pain.2009.06.001, indexed in Pubmed: 19592166.

23. Veves A, Manes C, Murray HJ, et al. Painful neuropathy and foot ulceration in diabetic patients. Diabetes Care. 1993; 16(8): 1187-1189, doi: 10.2337/diacare.16.8.1187, indexed in Pubmed: 8375249 .

24. Veves A, Young MJ, Manes C, et al. Differences in Peripheral and Autonomic Nerve Function Measurements in Painful and Painless Neuropathy: A clinical study. Diabetes Care. 1994; 17(10): 1200-1202, doi: 10.2337/diacare.17.10.1200.

25. Benbow SJ, Chan AW, Bowsher D, et al. A Prospective Study of Painful Symptoms, Small-fibre Function and Peripheral Vascular Disease in Chronic Painful Diabetic Neuropathy. Diabetic Medicine. 1994; 11(1): 17-21, doi: 10.1111/j.1464-5491.1994. tb00223.x.

26. Boulton AJM, Malik RA, Arezzo JC, et al. Diabetic somatic neuropathies. Diabetes Care. 2004; 27(6): 1458-1486, doi: 10.2337/ /diacare.27.6.1458, indexed in Pubmed: 15161806.

27. Spallone V, Greco C. Painful and painless diabetic neuropathy: one disease or two? Curr Diab Rep. 2013; 13(4): 533-549, doi: 10.1007/s11892-013-0387-7, indexed in Pubmed: 23677582.

28. Kramer HH, Rolke R, Bickel A, et al. Thermal Thresholds Predict Painfulness of Diabetic Neuropathies. Diabetes Care. 2004; 27(10): 2386-2391, doi: 10.2337/diacare.27.10.2386.

29. Vrethem M, Boivie J, Arnqvist H, et al. Painful Polyneuropathy in Patients With and Without Diabetes: Clinical, Neurophysiologic, and Quantitative Sensory Characteristics. Clin J Pain. 2002; 18(2): 122-127, doi: 10.1097/00002508-20020300000008.
30. Çakir N, Yetkin I, Karakoç A, et al. I-Carnitine in the treatment of painful diabetic neuropathy and its effect on plasma $\beta$-endorphin levels. Current Therapeutic Research. 2000; 61(12): 871-876, doi: 10.1016/s0011-393x(00)90014-6.

31. Tsigos C, Gibson S, Crosby SR, et al. Cerebrospinal fluid levels of beta endorphin in painful and painless diabetic polyneuropathy. J Diabetes Complications. 1995; 9(2): 92-96, doi: 10.1016/10568727(94)00024-i, indexed in Pubmed: 7599354.

32. Goodwin GM, Austin MP, Curran SM, et al. The elevation of plasma beta-endorphin levels in major depression. J Affect Disord. 1993; 29(4): 281-289, doi: 10.1016/0165-0327(93)90018-f, indexed in Pubmed: 8126314

33. Freeman R, Raskin P, Hewitt DJ, et al. CAPSS-237 Study Group. Randomized study of tramadol/acetaminophen versus placebo in painful diabetic peripheral neuropathy. Curr Med Res Opin. 2007; 23(1): 147-161, doi: 10.1185/030079906X162674, indexed in Pubmed: 17257476.

34. Veening JG, Gerrits PO, Barendregt HP. Volume transmission of beta-endorphin via the cerebrospinal fluid; a review. Fluids Barriers CNS. 2012; 9(1): 16, doi: 10.1186/2045-8118-9-16, indexed in Pubmed: 22883598.

35. Skipor J, Thiery JC. The choroid plexus œ cerebrospinal fluid system: Undervaluated pathway of neuroendocrine signaling into the brain. Acta Neurobiol Exp. 2008; 68: 414-428.

36. Foley KM, Kourides IA, Inturrisi CE, et al. beta-Endorphin: analgesic and hormonal effects in humans. Proc Natl Acad Sci U S A. 1979; 76(10): 5377-5381, doi: 10.1073/pnas.76.10.5377, indexed in Pubmed: 291954.

37. Butelman ER, Reed B, Chait BT, et al. Limited effects of beta-endorphin compared to loperamide or fentanyl in a neuroendocrine biomarker assay in non-human primates. Psychoneuroendocrinology. 2008; 33(3): 292-304, doi: 10.1016/j.psyneuen.2007.11.011, indexed in Pubmed: 18171605.

38. Bruehl S, Chung OkY, Chont M. Chronic pain-related changes in endogenous opioid analgesia: a case report. Pain. 2010; 148(1): 167-171, doi: 10.1016/j.pain.2009.09.025, indexed in Pubmed: 19833435.

39. Jin HY, Lee KAe, Park TS. The impact of glycemic variability on diabetic peripheral neuropathy. Endocrine. 2016; 53(3): 643-648, doi: 10.1007/s12020-016-1005-7, indexed in Pubmed: 27383181.

40. Xu F, Zhao LH, Su JB, et al. The relationship between glycemic variability and diabetic peripheral neuropathy in type 2 diabetes with well-controlled HbA1c. Diabetol Metab Syndr. 2014; 6(1): 139, doi: 10.1186/1758-5996-6-139, indexed in Pubmed: 25530811.

41. Oyibo SO, Prasad Y, Jackson NJ, et al. The relationship between blood glucose excursions and painful diabetic peripheral neuropathy: a pilot study. Diabetic Medicine. 2002; 19(10): 870-873, doi: 10.1046/j.1464-5491.2002.00801.x.

42. Padilla A, Descorbeth M, Almeyda AL, et al. Hyperglycemia magnifies Schwann cell dysfunction and cell death triggered by PA-induced lipotoxicity. Brain Res. 2011; 1370: 64-79, doi: 10.1016/j.brainres.2010.11.013, indexed in Pubmed: 21108938.

43. Kassem HS, Azar ST, Zantout MS, et al. Hypertriglyceridemia and peripheral neuropathy in neurologically asymptomatic patients. Neuro Endocrinol Lett. 2005; 26(6): 775-779, doi: 10.1016/ /b978-0-7216-9491-7.x5001-7, indexed in Pubmed: 16380709. 